\title{
X-RAY ABSORPTION STUDY OF Ti, Cu AND Fe IMPLANTED AIN
}

\author{
M. Borowski, A. Traverse \\ LURE, Univ. Paris Sud, Bât. 209 A, 91405 Orsay, France
}

AND J. MimaulT

Faculté des Sciences, Lab. de Métallurgie Physique, 86022 Poitiers, France

Sintered AlN ceramics were implanted by $\mathrm{Ti}, \mathrm{Fe}$ and $\mathrm{Cu}$ ions up to $1.9 \times 10^{17}$ atoms $/ \mathrm{cm}^{2}$ at mean energies of $70-110 \mathrm{keV}$ in order to investigate the role of the chemical properties of the implanted species. on the phase formed during the implantation process. X-ray absorption studies were performed at room and at liquid nitrogen temperature to give information on the resulting systems and local environments of the $\mathrm{Ti}, \mathrm{Fe}$ and $\mathrm{Cu}$ atoms. We observe the formation of TiN even for as-implanted samples, while the $\mathrm{Cu}$ ions aggregate to clusters. The Fe implanted samples show an intermediate behavior with both nitride formation and Fe clustering. In conclusion, the heat of formation is found to be a key parameter for the final system.

PACS numbers: 81.60.Dq, 61.10.Lx, 61.80.Jh

\section{Introduction}

The excellent thermal conductivity and good electrical isolation, in addition with a thermal expansion coefficient close to that of silicon $[1,2]$ makes AlN the most promising candidate as a support in VLSI technology. The metallization of the ceramic surfaces is an important question for this application. A technique to modify the surface is ion implantation. In the collision cascade initiated by the implanted ion, the system is in a quasi-liquid state for $10^{-11}-10^{-10} \mathrm{~s}$ [3], during which chemical bonds are cracked. The question is how the atoms will rearrange after the cascade. As implantation is far from thermodynamical equilibrium process, one can ask about the role of the thermodynamical parameters, namely the heats of formation of possible compounds $[4,5]$, on the final systems. In the case of $\mathrm{Cu}$ implanted in AlN $[6,7]$, the implanted metal was found to form small clusters with fcc structure and a lattice parameter close to the bulk one. The heat of formation for $\mathrm{Cu}$-nitrides is positive $\Delta H_{f^{0}}=17.8 \mathrm{kcal} / \mathrm{mol}$ and $\Delta H_{f^{0}}=66.7 \mathrm{kcal} / \mathrm{mol}$ 
for $\mathrm{Cu}_{3} \mathrm{~N}$ and $\mathrm{CuN}_{3}$ [8], respectively, like the one of the intermetallic compound. What phase will be formed, if the heat of formation is close to the matrix one? For example, $\Delta H_{f^{u}}=-80.8 \mathrm{kcal} / \mathrm{mol}$ for TiN is comparable to the heat of AlN formation $\Delta H_{f^{\prime}}=-76.0 \mathrm{kcal} / \mathrm{mol}$, while $\Delta H_{f^{\prime \prime}}=-2.6$ for $\mathrm{Fe}_{4} \mathrm{~N}$ formation (at $298 \mathrm{~K}[8]$ ). Hence, irnplantation of $\mathrm{Ti}$ and $\mathrm{Fe}$ in AlN seerns to offer the possibility to answer this question.

To characterize the resulting system and phase formation, we used X-ray absorption spectroscopy (XAS). The near edge region of the absorption spectra (XANES, X-ray absorption near edge structure) is strongly influenced by the chernical environment of the probe atom, while the interaction of the ernitted $K$-electron with the surrounding atoms yields oscillations that extend up to $1000 \mathrm{eV}$ from the edge (EXAFS, extended X-ray absorption fine structure). The Fourier transformation of these oscillations represents a pseudo-radial distribution of the mean surrounding of the element. By simulation of the oscillations one can determine the kind of neighbors and their average number and distances.

\section{Experimental}

AlN ceramic bulk samples (purchased by Kerannont) were implanted with fluences and energies given in Table I. Ti was implanted with one energy leading to a Gaussian distribution of the implanted species. Implantations of $\mathrm{Cu}$ and $\mathrm{Fe}$ were done at two different fluences and at two energies to obtain a more homo-

TABLE I

Implantation parameters for the different samples.

\begin{tabular}{c|c|r|c}
\hline \hline Ion & $\begin{array}{c}\text { Fluence } \phi \\
{\left[10^{16} \text { at. } / \mathrm{cm}^{2}\right]}\end{array}$ & $\begin{array}{c}E \\
{[\mathrm{keV}]}\end{array}$ & $\begin{array}{c}\text { Conc. } \\
{[\mathrm{at} . \%]}\end{array}$ \\
\hline $\mathrm{Ti}$ & 10.0 & 70 & 14 \\
\hline $\mathrm{Fe}$ & 5.8 & 80 & 10 \\
& 0.8 & 20 & \\
$\mathrm{Fe}$ & 11.5 & 80 & 20 \\
& 1.5 & 20 & \\
$\mathrm{Fe}$ & 16.5 & 80 & 30 \\
& 2.2 & 20 & \\
\hline $\mathrm{Cu}$ & 5.0 & 100 & 7 \\
$\mathrm{Cu}$ & 0.6 & 30 & \\
& 10.0 & 100 & 14 \\
$\mathrm{Cu}$ & 1.2 & 30 & \\
& 14.3 & 100 & 20 \\
& 1.7 & 30 &
\end{tabular}


geneous profile up to a depth of about $50 \mathrm{~nm}$. In the case of $\mathrm{Fe}$, a fraction of the implanted ions was ${ }^{57} \mathrm{Fe}$ to give the possibility of complementary studies by the Mössbauer spectroscopy. To avoid target heating the samples were water cooled and the implantation current was limited, so that the power transferred to the sample did not exceed $100 \mathrm{~mW} \mathrm{~cm}^{-2}$.

To recover the AIN matrix from defects introduced during implantation and to study the influence of annealing on the implanted species, some of the samples were kept at $800^{\circ} \mathrm{C}$ for $1 \mathrm{~h}$ to $5 \mathrm{~h}$ under vacuum $\left(<10^{-7}\right.$ torr $)$.

Rutherford backscattering spectroscopy (RBS) with $1.2 \mathrm{MeV}$ single charged He-ions was performed on the samples to cletermine the depth profile of the implanted ions. In the case of $\mathrm{Cu}, 10 \%$ to $20 \%$ of the total amount is lost after annealing, while all $\mathrm{Ti}$ and $\mathrm{Fe}$ atoms are retained. The profiles of the last two species are slightly sharper. The accelerators used for implantation and analysis are described elsewhere [9]. X-ray absorption spectra were taken at. DCI beam line of the LURE synchrotron, either at the XAS 3 experiment for the Ti-implanted samples using Si (311) double-crystal monochromator, or at the XAS 1 experiment, equipped with a $\mathrm{Si}$ (331) channel cut monochromator, for $\mathrm{Cu}$ and $\mathrm{Fe}$ edge. Measurements were performed in the total electron detection mode. The detection range limited by the mean free path of the electrons, is typically of the order of $100 \mathrm{~nm}$ and therefore adapted to the study of the implantation depth range.

Spectra were taken at room temperature at the $4966 \mathrm{eV} \mathrm{K}$-absorption edge of $\mathrm{Ti}$ in $1 \mathrm{eV}$ steps in the XANES region and $2 \mathrm{eV}$ for EXAFS. For Fe and $\mathrm{Cu}$ implanted sarnples, we worked at $80 \mathrm{~K}$ at the $K$-absorption edge of the two elements at $7112 \mathrm{eV}$ and $8979 \mathrm{eV}$, respectively, using $2 \mathrm{eV}$ energy steps for EXAFS and $0.5 \mathrm{eV}$ in the XANES region.

During the Ti experiments, a TiN sample of well-known composition and lattice parameter [10], a $\mathrm{TiO}_{2}$ rutile and a pure $\mathrm{Ti}$ sample were measured as standards. In the case of $\mathrm{Fe}$ and $\mathrm{Cu}$, we used the metallic foils and a $\mathrm{Fe}_{3+w} \mathrm{~N}$ sample as standards.

\section{Results}

\section{1. $A l N / T i$}

The profile of the implanted $\mathrm{Ti}$ ions was found to correspond to a Gaussian distribution at a depth of $32 \mathrm{~nm}$ with a FWHM of $52 \mathrm{~nm}$. In Fig. 1 the XANES spectrum of the annealed AlN/Ti sarnple is similar to the TiN 0.96 standard. The spectrum of the as-implarited AlN/Ti is different from both Ti and $\mathrm{TiN}_{0.96}$ standard. There is no detectable contribution of oxidation. The EXAFS analysis on annealed AIN/Ti confirms the previous conclusion. A fitting was done by using phases and amplitudes deduced from $\mathrm{TiN}_{0.96}$ with 6 first $\mathrm{N}$ neighbors at $2.121 \AA$ and 12 second Ti neighbors at $2.999 \AA$. This leads in AlN/Ti to 5.3(8) first $\mathrm{N}$ neighbors at 2.114(12) $\AA$ and 12.0(13) second Ti neighbors at 3.004(5) $\AA$.

For the as-implanted system, the weighted Fourier transform in Fig. 2 already shows a first $\mathrm{N}$ shell, which could be fitted by $3 \mathrm{~N}$ at 1.98(8) $\hat{\AA}$. At larger distances, the disorder is too large, therefore no second shell could be identified. 


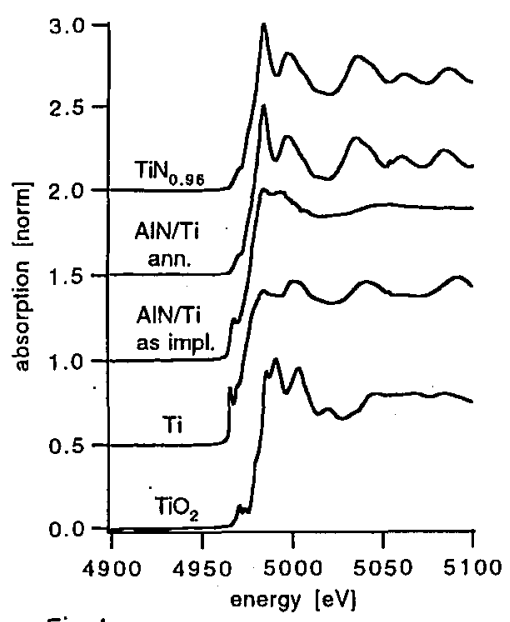

Fig. 1

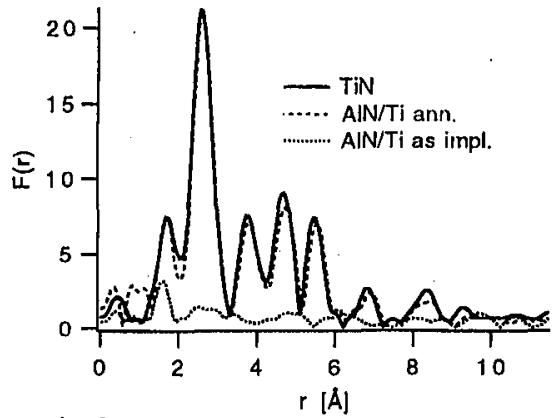

Fig. 2

Fig. 1. Structure near the $\mathrm{Ti} K$-edge of the implanted samples, pure $\mathrm{Ti}, \mathrm{TiO}_{2}$ and $\mathrm{TiN}_{0.96}$.

Fig. 2. Fourier transform of the implanted samples and the $\mathrm{TiN}_{0.96}$ standard.

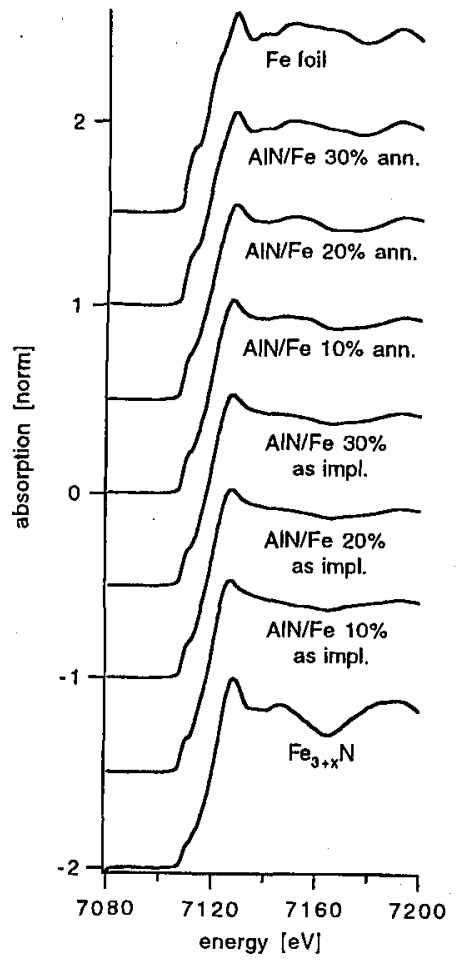

Fig. 3. XANES spectra of Fe implanted AIN samples. 


\section{2. $A l N / F e$}

The near edge structure in Fig. 3 of the annealed sample with 30 at.\% average concentration is quite similar to the pure Fe spectrum. For the annealed samples with smaller amount of $\mathrm{Fe}$ one recognizes a smoothing of the features and a minimum at around $7160 \mathrm{eV}$ appears. All as-implanted spectra are rather smooth, but they have only small similarity with the spectrum of the $\mathrm{Fe}$ foil. In comparison to the annealed samples, the minimum at around $7160 \mathrm{eV}$ is more accentuated. As such minimum is also present in the XANES of $\mathrm{Fe}_{3+x} \mathrm{~N}$, there is a possibility of $\mathrm{Fe}-\mathrm{N}$ bonds.

The Fourier transforms show a similar behavior. Only for the highest concentration we can simulate the EXAFS oscillation with pure Fe. While pure Fe has 8 next neighbors, we find a value of 7.6 at distances corresponding to the Fe bulk. This corresponds to a cluster size greater than $60 \AA$. For the other samples it is possible to improve the fit by adding a $N$ contribution, but reliable quantitative treatment is rather difficult.

\section{3. $\mathrm{AlN} / \mathrm{Cu}$}

The Fourier transform shows for all the $\mathrm{Cu}$-implanted samples a typical fcc-like spectrum as shown in Fig. 4. Only for the smallest concentration one observes the existence of disorder at larger distances. For the other concentrations the spectra are similar to pure $\mathrm{Cu}$ even at larger distances. The decrease in the peak amplitude, due to the reduction of the next neighbor numbers depending on the size of the cluster, is well demonstrated. By computing a fcc cluster of growing size, we calculated the average number of first neighbors, $N$, versus the cluster diameter, $D$, hence to relate the experimental $N$ value to $D$. The results are given in Table II. The first neighbor distance in the clusters is found to be close to the bulk one.

For the smallest concentration, hence for the smallest cluster size, we can see the disappearance of the $\beta$ peak in the near edge structure (Fig. 5) [11].

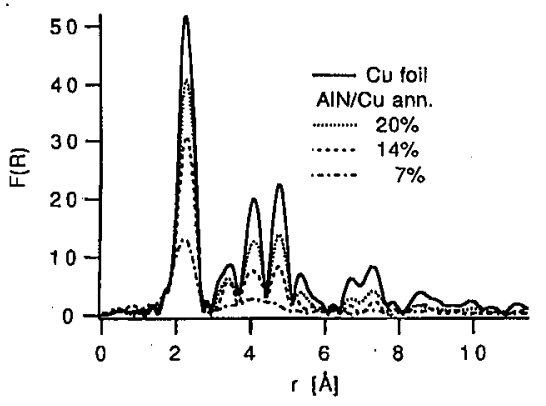

Fig. 4. Fourier transform of the implanted $\mathrm{Cu}$ samples in comparison with a $\mathrm{Cu}$ foil. 
TABLE II

$N$ is the experimental average number of first neighbors at a distance $d$ and $D$ is the diameter of the Cu cluster.

\begin{tabular}{c|c|c|c}
\hline \hline Sample & $N$ & $d[\AA]$ & $D[\AA]$ \\
\hline $7 \%$ ann. 1 h & 6.5 & 2.53 & 8.5 \\
$7 \%$ ann. 5 h & 7.7 & 2.53 & 10 \\
$14 \%$ as-impl. & 9.2 & 2.54 & 16 \\
$14 \%$ ann. 5 h & 9.9 & 2.55 & 21 \\
$20 \%$ as-irripl. & 8.9 & 2.55 & 13 \\
$20 \%$ ann. 5 h & 10.7 & 2.55 & 36 \\
foil & 12 & 2.55 &
\end{tabular}

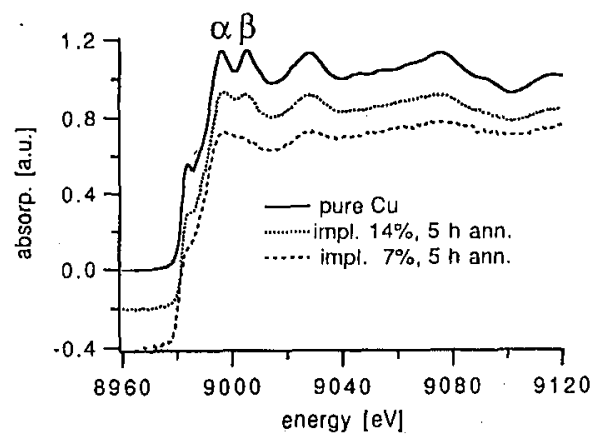

Fig. 5. XANES spectra for small Cu concentrations in the implanted samples.

\section{Conclusion}

For Ti implantation, short range order (on a scale of about $2 \AA$ ) is achieved in the cascade, due to the trapping of $\mathrm{N}$ by $\mathrm{Ti}$. In the case of $\mathrm{Cu}$, metallic clusters in AlN can be formed. Their size can be controlled by the implantiation fluence and the thermal treatment. By means of X-ray absorption the size of the aggregates can be determined in addition to the local environment.

The formation of TiN and the clustering of the implanted $\mathrm{Cu}$ ions indicate that the heat of formation is a key parameter to predict the nature of the final systerns produced by ion implantation.

This conclusion is in agreement with the results of Fe implantation. Although EXAFS analysis alone is not able to deterrnine the contributions of the different phases in a reliable quantitative way, one can see that the AlN/Fe system is an intermediate situation between the two other systems, as predicted by the heats of formation. Other experiments like the Mössbauer spectroscopy will be performed to identify the phases formed in addition to the Fe clusters. 


\section{Acknowledgment}

We would like to thank the ARAMIS/IRMA staff at the Centre de Spectrométrie Nucléaire et de Spectrométrie de Masse (CSNSM) at Orsay for the help during the accelerator experiments, especially O. Kaitasov for the sample implantations. This work was partially supported (M.B.) by a grant from the DAAD (German academic exchange service) HSP II program.

\section{References}

[1] L.M. Sheppard, Am. Ceram. Soc. Bull. 70, 1467 (1991).

[2] D.D. Marchant, T.E. Nemecek, Am. Ceram. Soc., Adv. Ceram. 26, 19 (1989).

[3] R..S. Averback, T. Diaz de la R.ubia, Horngming Hsieh, R. Benedek, Nucl. Instrum. Methods Phys. Res. B 59/60, 709 (1991).

[4] Y.T. Cheng, Mater. S'ci. Rep. 5, 45 (1990).

[5] J. Desimoni, A. Traverse, Phys. Rev. B 48, 13266 (1993).

[6] J. Du, Ph.D. Thesis, Université de Paris XI, 1992.

[7] J. Du, A. Traverse, S. Hagége, Mater. Sci. Forum B 126/128, 703 (1993).

[8] D.D. Wagman, W.H. Evans, V.B. Parker, R.H. Schumm, S.M. Bailey, I. Halow, K.L. Churney, R.L. Nuttall, in: CRC-Handbook of Chemistry and Physics, Eds. R.C. Weast, M.J. Astle, CRC Press, Boca Raton 1989, D-50.

[9] H. Bernas, J. Chaumont, E. Cottereau, R. Meunier, A. Traverse, C. Clerc, O. Kaitasov, F. Lalu, D. Le Du, G. Moroy, M. Salomé, Nucl. Instrum. Methods Phys. Res. B 9, 416 (1992).

[10] W. Lengauer, J. Alloys Compounds 186, 293 (1992).

[11] A. Traverse, P. Parent, J. Mimault, S. Hagège, J. Du, Nucl. Instrum. Methods Phys. Res. B 84, 204 (1994). 